\title{
Is Bariatric Surgery Effective in Reducing Comorbidities and Drug Costs?: Letter to the editor
}

\author{
Ina Gesquiere $^{1}$ - Judith Aron-Wisnewsky ${ }^{2}$
}

Published online: 7 January 2016

(C) Springer Science+Business Media New York 2016

Dear editor,

We have read with great interest the article "Is bariatric surgery effective in reducing comorbidities and drug costs? A systematic review and meta-analysis," by Lopes and colleagues [1]. Although this study has compiled a lot of studies and included a large number of patients, we have some questions concerning the methodology. The PICO strategy was used to construct the methodology search for this systematic review, and the authors followed the PRISMA guidelines to conduct a literature review. However, we think that if more synonyms of the keywords had been added to the search strategy, the authors would have found and included more articles in this literature review therefore increasing the power of the results they herein present. For example, different terms for drug cost and pharmacoeconomics were implemented, but the term "medication cost" was not included. If this term had been added to the strategy search, articles meeting the inclusion criteria, such as "Medication cost is significantly reduced after Roux-en-Y Gastric Bypass in obese patients" and "Short-term medication cost savings for treating hypertension and diabetes after gastric bypass" would have also been included in this

Ina Gesquiere

ina.gesquiere@pharm.kuleuven.be

1 Clinical and Experimental Endocrinology, KU Leuven and Department of Endocrinology,University Hospitals Leuven/KU Leuven, O\&N1, Herestraat 49, box 902, 3000 Leuven, Belgium

2 Institute of Cardiometabolism and Nutrition (ICAN), Assistance Publique-Hôpitaux de Paris, INSERM, Sorbonne Université, Paris 6, Pitié-Salpêtrière Hospital, F-75013 Paris, France systematic review and meta-analysis [2, 3]. The results of these two published studies are in line with the other papers discussed in this systematic review. Therefore, we do not believe that including these papers would have changed the overall conclusion of this meta-analysis. However, it would have given a more comprehensive vision of what has already been published. Indeed these two studies have included quite an important number of patients, comparable to that of some of the studies already present in this meta-analysis. Overall, we believe that a more exhaustive search strategy could have yet improved this already extensive paper and strengthen its impact.

Yours sincerely.

\section{Compliance with Ethical Standard}

Conflict of Interest The authors declare that they have no competing interests.

\section{References}

1. Lopes EC, Heineck I, Athaydes G, et al. Is bariatric surgery effective in reducing comorbidities and drug costs? A systematic review and meta-analysis. Obes Surg. 2015;25:1741-9.

2. Gesquiere I, Aron-Wisnewsky J. Medication cost is significantly reduced after Roux-en-Y Gastric Bypass. Obes Surg. 2014;24(11): 1896-903.

3. Ghiassi S, Morton J, Bellatorre N, et al. Short-term medication cost savings for treating hypertension and diabetes after gastric bypass. Surg Obes Relat Dis. 2012;8(3):269-74. 